\title{
Prácticas de mercadeo en instituciones de educación superior en el contexto de la globalización
}

\section{Marketing practices in institutions of higher education in the context of globalization}

\author{
Estefanía Montoya Botero (Colombia) \\ Especialista en gerencia de marketing \\ Universidad Pontificia Bolivariana \\ montoya.botero.estefania@gmail.com
}

\section{Resumen}

Esta investigación tiene un enfoque cualitativo-exploratorio sustentado en la revisión bibliográfica y el análisis documental de las experiencias publicadas sobre Sistemas de Información de Mercadeo y dirección estratégica de mercadeo educativo, en antecedentes encontrados en bases de datos académicas como Scopus y Procuest con el fin de obtener un insumo de las mejores prácticas de mercadeo para el diseño del SIM de la Universidad Pontificia Bolivariana. Sin embargo, después del análisis realizado a los documentos descargados, se evidenció que actualmente las universidades no tienen estructurado el proceso administrativo de comercialización del servicio de educación superior en un SIM, sino más

\section{Summary}

This research has a qualitative-exploratory approach, supported in bibliographic review and documentary analysis of marketing information systems and educational marketing strategic management published experiences, as in academic databases found antecedents such as Scopus and Procuest, in order to obtain a best marketing practices input for Universidad Pontificia Bolivariana SIM design. However, after analyzing the downloaded documents, it was evident that universities currently do not have structured any SIM administrative process of higher education service marketing, but
Recibido:

Evaluado:

Aceptado:
8 de abril de 2016

15 de enero de 2017

3 de abril de 2017

\section{PARA CITAR ESTE ARTíCULO/TO CITE THIS ARTICLE}

Montoya Botero, E. (2017). Prácticas de mercadeo en instituciones de educación superior en el contexto de la globalización, Poliantea 13(24), pág ?ִ??-??. 
bien utilizan diferentes estrategias como la Orientación al mercado, estrategias de CRM, o Gestión de marca para vender el servicio educativo. Por tal motivo, las evidencias encontradas incentivan la profundización de investigación en este tema y abren la puerta al debate conceptual frente a las estrategias y herramientas utilizadas en la comercialización del servicio educativo.

El impacto social de esta investigación enfatiza la responsabilidad de las Universidades en formar integralmente a las personas que la constituyen buscando siempre el progreso y mejoramiento continuo de los procesos de gestión administrativa institucional en pro de la cooperación directa en el desarrollo de seres de cultura y sociedad.

Palabras Clave: Prácticas de mercadeo, globalización, Sistema de Información de Mercadeo, Dirección de mercadeo, Mercadeo de servicios educativos. they rather use different strategies such as market orientation, CRM strategies or brand management to sell their educational service. Therefore, found evidences encourage into this subject research deepening, and open the door to conceptual debate regarding educational service commercialization used strategies and tools.

This research social impact emphasizes Universities responsibility to comprehensively train their people, always seeking institutional administrative management progress and continuous improvement in favor of direct cooperation in the development of culture and society persons.

Keywords: Marketing practices, globalization, Marketing Information System, Marketing Management, Educational Services Marketing. 


\section{Introducción}

La aplicación del mercadeo en las instituciones de educación superior, como las universidades, obedece a diferentes estructuras; algunas, han constituido una unidad encargada de la función del mercadeo bajo unidades organizativas que siguen objetivos de promoción y soporte estratégico; otras se ciñen a la aplicación de herramientas e instrumentos sin definir explícitamente un órgano o unidad de mercadeo. No obstante, la incertidumbre y fluctuación que se desata con el fenómeno de la globalización enmarca para este tipo de organizaciones la necesidad de restructurar y entender el mercadeo como un aliado para dar respuesta a las necesidades sociales y a su vez modificar la oferta educativa que consulte dichas necesidades.

En este contexto, se hace imperante estructurar la gestión y un sistema de flujo de información de mercadeo que contribuya al proceso de toma de decisiones de manera trasversal a toda la organización. Esta estructura responde a la denominación de un Sistema de Información de Mercadeo (Caetano, 2003;
Naranjo, 2011 y Ponce, 2008) que permite recoger, almacenar y distribuir información externa e interna a la organización favoreciendo el proceso gerencial (Romeiro y Garmendia, 2007; Kotler y Keller, 2012 y Hernández, 2003).

Basados en un análisis documental, como primera etapa de una investigación más amplia, se propuso revisar y analizar los antecedentes bibliográficos en bases de datos académicas con el fin de identificar las mejores prácticas de universidades que cuentan con una estructura organizativa de mercadeo. No es posible desde la información analizada identificar un Sistema de Información de Mercadeo como una estrategia notable dentro del trabajo de gestión de mercadeo de las instituciones de educación superior. Pero si es posible destacar el uso de herramientas como los procesos de orientación al mercado, la implementación de programas de relacionamiento con el cliente Consumer Relationship Management - CRM $-y$ la gestión de marca.

Para el caso colombiano las instituciones de educación consultadas afir- 
man no tener un Sistema de Información de Mercadeo - SIM - propiamente estructurado, no obstante, cuentan con departamentos o áreas funcionales de mercadeo que gestiona e impulsan comercialmente la oferta de programas educativos.

Debido a que la oferta de programas de pregrado y postgrado está altamente dominado por instituciones de educación superior de carácter privado en Colombia, su fuente de ingresos fundamentalmente proviene de las matrículas de los estudiantes lo que obliga a la administración de éstas instituciones a desarrollar unidades de procesos y planes de mercadeo en ocasiones no explícitos pero que básicamente persiguen el objetivo de atraer y mantener a los estudiantes dentro de estos programas. Bajo este esquema, integrar un Sistema de Información de Mercadeo a la gestión integral estratégica contribuye a comprender y gestionar una función esencial de la administración con las particularidades y necesidades propias de las universidades colombianas en relación con el contexto global.

\section{Marco teórico}

\section{Globalización y educación superior}

El fenómeno de la globalización emergente en las últimas décadas modificó el intercambio económico y mercantil entre las naciones, y favoreció la renovación del estilo de compra y el nivel de exigencias que un cliente demanda de una marca. La globalización no solo se define como una teoría de comportamiento económico, sino también como una realidad social que aqueja actualmente a todos los mercados, cambiando por completo las estrategias financieras de las naciones $y$ redefiniendo conductas comportamentales y patrones de cultura, transformando definitivamente el progreso de los países en vía de desarrollo. (Romero, 2002, citado en León, 2004, p.347) (Mateus y Brasset, 2002).

El impacto de la aparición de este fenómeno fue tan trascendental, que el mercado se volcó a la competencia internacional y originó "nuevos procesos productivos, distributivos y de consumo deslocalizados geográficamente; logrando no solo una expansión y uso intensivo de la tecnología" (Mateus y Brasset, 2002, p.67) sino también a que las empresas se enfrentaran a las nuevas exigencias de un cliente más informado y a un mercado más riguroso en términos competitivos de ciencia y tecnología aplicada (León, 2004).

La globalización cambió el mundo y necesariamente se debe replantear el papel fundamental que cumple la educación, pues desde este parámetro, un país puede generar la apertura intelectual que requiere para competir en el mercado global. "Las ventajas comparativas de los países depende de capacidades y ventajas adquiridas, con base en el desarrollo de recursos humanos y en la capacidad para generar y aplicar conocimiento, tanto en la producción como en la solución de problemas sociales" (León, 2004, p.349).

La ciencia útil es la llave de inserción al mundo aguerrido y competido de las 
empresas globales del siglo XXI que se orienten a la lógica del capital y del mercado, a la búsqueda de la ganancia y la alta productividad. "El mundo moderno reclama una educación de calidad, con pertinencia e internacionalizada... Una Universidad de excelencia, ubicada en el escenario mundial y en la modernidad pensando con profundidad en su misión y su visión frente a las especificidades del entorno en el cual se asienta" (León, 2004, p.349).

\section{La educación internacional}

Para Milton Ricardo Ospina y Pedro Emilio Sanabria investigadores de la Universidad Militar Nueva Granada, la educación es uno de los pilares fundamentales del desarrollo de cualquier sociedad y tiene como objetivo principal la formación de ciudadanos íntegros que contribuyan al desarrollo de la sociedad misma convirtiendo así a las universidades en el motor principal del avance social. (Ospina y Sanabria, 2010) Sin embargo, esta perspectiva educativa cambió gracias a la nueva configuración mundial surgida en las últimas décadas, abriendo camino a la interculturalidad mundial y proponiendo un nuevo sistema de mercadeo global.

Tradicionalmente las organizaciones de carácter educativo se desarrollaron en un medio estable y protegido durante mucho tiempo, sin embargo, en los últimos años el entorno se ha transformado dramáticamente llevando a cambios significativos en el tipo de formación que la sociedad demanda del sistema educativo de hoy. (Ospina y Sanabria, 2010, p.117)
Por ello, las instituciones universitarias se convirtieron en empresas prestadoras de servicios educativos desdibujando la idea de universidad de élite y proponiendo la universidad de masas, favoreciendo un ambiente de creciente competitividad y diversificación a escala nacional e internacional de la oferta. Adicional esta propuesta, logró también que tanto la comunidad universitaria, como los gobiernos y los expertos en educación superior, propusieran que los sistemas universitarios actuales se orienten al mercado, creando un fenómeno de "comercialización" a escala mundial. (Ponce, 2008) (Hemsley y Oplatka, 2006) (Laval, 2004).

El mercadeo de las universidades incentiva la búsqueda continua de diferenciación de oferta y proporciona una oportunidad clara para la mejora en la gestión administrativa en pro de la adaptación al cambio y responder así a los desafíos que se le presentan hoy. (Ponce, 2008) (Caetano, 2003) Actualmente, "las instituciones de enseñanza frecuentemente encuentran dificultades para mantener la lealtad de sus clientes frente a numerosos factores concurrentes como las características innovadoras de nuevos cursos, mejores instalaciones, disponibilidad e innovaciones tecnológicas aplicadas al sector" (Caetano, 2003, p.16).

\section{La universidad internacional}

Desde la época de Aristóteles, entre el siglo XII y el siglo XIII, la Universidad nació como institución educativa dedicada a la formación de personal cualificado para el servicio de la Iglesia y el 
Estado. Dese allí, la Universidad evoluciona cada día en su ejercicio mismo, y se adapta en pro del mejoramiento continuo de sus facultades, con el fin de conferir grados académicos correspondientes con las exigencias y necesidades de la sociedad y el mercado contemporáneo que las enfrenta a la reinvención y adaptación al clima globalizado del conocimiento y la ciencia actual. (Real Academia Española, 2015) (Théry, 2013).

Según El Ministerio de Educación Nacional de la República de Colombia (2009), la internacionalización de las instituciones de educación superior trae consigo muchos beneficios:

Facilita la inserción de estudiantes en un mundo globalizado y permite un mayor intercambio de conocimientos, transferencia de tecnologías e investigación; brinda la posibilidad de incrementar la movilidad de estudiantes, profesionales, docentes e investigadores; propicia el mejoramiento de los estándares de acreditación y la armonización de los criterios con que se evalúa la calidad de los programas académicos y las instituciones en diferentes países; y por último, facilita la expansión de servicios de enseñanza por parte de las Instituciones de Educación Superior (Ministerio de Educación Nacional, 2009).

Fue entonces cuando la internacionalización de la educación se convirtió en un distintivo de calidad y de diferenciación de la oferta académica en el medio, otorgándole presencia y visibilidad internacional a las instituciones ed- ucativas en un mundo cada vez más globalizado. Por su parte, la Universidad de León en España desde hace 4 años expuso la importancia de iniciar un proceso de internacionalización para su institución y lograr así una característica diferenciadora para su comercialización.

\begin{abstract}
Una universidad que facilita y apoya la colaboración internacional, a la par que la movilidad de su personal y de sus estudiantes podrá competir con más eficacia en el mercado internacional para atraer estudiantes e investigadores de alto nivel, para obtener fondos de investigación y financiación de programas internacionales y para dotar a sus estudiantes de un necesario bagaje lingüístico, cultural y formativovivencial que les posibilitará enfrentarse posteriormente a los retos de la sociedad actual globalizada con mayor garantía de éxito (Universidad de Léon, 2012, p.1).
\end{abstract}

Pero, ¿Cómo puede una Universidad iniciar un proceso de internacionalización? después de evaluar ligeramente el contexto global que viven a diario las universidades alrededor del mundo, es importante enfatizar, que todo este esfuerzo de articulación institucional requiere de una planeación estratégica enfocada en la buena comercialización de la marca, reconociendo desde el principio la capacidad de respuesta de la Universidad a las exigencias del mercado actual, realizando un diagnóstico previo del funcionamiento de la institución día a día, de la capacidad de adaptación de los sistemas administrativos y burocráticos de la Universidad al entorno global, 
la posibilidad de desarrollar nuevas habilidades y promover la agilidad en los procesos administrativos y trámites financieros para poder soportar el posible incremento de la afluencia de nuevos clientes a la institución (Universidad de Léon, 2012).

Para Ximena C. Arango Directora del Departamento de Relaciones Internacionales de la U.C.D.A "En el contexto de la Educación Superior, la internacionalización es un fenómeno multifacético omnipresente en toda la institución universitaria, que toca cada aspecto de sus directrices". (Arango, 2009 , p.1) Se afirma además que dentro del proceso de internacionalización de una institución, es importante conocer los niveles profundamente relacionados dentro del funcionamiento normal de la misma, pues este proceso "se caracteriza por ser un proceso de transformación en el cual las áreas de actividad de una institución son constantemente adecuadas para operar en un medio internacional" (The Danish Rectors' Conference, 2000, citado en Arango, 2009, p.3).

\section{Articulación de la Universidad con la In- ternacionalización y la Comercialización}

Si bien es claro, el ambiente globalizado del mundo ha inmerso a las Universidades en una competencia sin precedentes; "las ha obligado a gastar más en la contratación y comercialización de sus marcas" (Shaver, 2012, p.introductoria) redefiniendo completamente el plan estratégico de operación y comercialización de las instituciones educativas modernas. (Williams y
Omar, 2014; Wang, 2009; Shaver, 2012)

Hoy por hoy los dirigentes universitarios son impulsados principalmente por motivos educativos, pero factores económicos están convirtiendo rápidamente a las fuerzas igualmente poderosas. Aunque la mayoría de las Universidades se definen como organizaciones sin ánimo de lucro, sólo pueden seguir existiendo si suficientes estudiantes continúan eligiendo esa Universidad y luego persisten en su elección. Por tal motivo, y debido al cambio económico que tuvo la sociedad a raíz de la apertura de los mercados a nivel mundial, las instituciones educativas entendieron que no solo la internacionalización de sus currículos iba a generar los recursos necesarios para su subsistencia, sino que también debían poner en práctica diferentes planes de contingencia para retener y atraer al mismo tiempo la mayor cantidad de estudiantes a su plantel educativo, convirtiendo así a la globalización y diversidad de oferta en una aliado estratégico para la comercialización de su marca a nivel global. (Shaver, 2012; Wang, 2009; Lombao, 2006)

Dentro de los estudios referentes para esta investigación se encontraron ejemplos de cómo las Universidades a nivel mundial, han empezado a trabajar fuertemente en la relación de su marca con su cliente directo. Para Benjamin J. Shaver (2012)

Tradicionalmente, las empresas han concentrado sus esfuerzos de mercadeo en atraer a nuevos clientes para maximizar los beneficios, pero durante las últimas décadas, las em- 
presas orientadas a los servicios se han desplazado de esta estrategia de mercadeo tradicional hacia un enfoque "relationship-marketing" que se centra en el desarrollo de relaciones a largo plazo con los clientes existentes (Barnes, 1994, citado en Shaver, 2012, p.1). Este enfoque asume que la retención de clientes satisfechos en última instancia resultar más rentable que gastar continuamente presupuesto de mercadeo en la obtención de nuevos clientes. (Shaver, 2012, p.1-3)

Y no solo eso, se han dado cuenta de que retener a los estudiantes actuales es en realidad una estrategia de afiliación más rentable que simplemente reclutarlos. Pues desarrollar un fuerte vínculo entre la Universidad y los estudiantes no solo beneficia monetariamente a la institución durante un plazo, sino que también puede generar beneficio después de la graduación de los estudiantes pues "ex alumnos leales son propensos a promover la Universidad a los futuros estudiantes, dar donaciones, y tal vez volver a su alma mater para la escuela de posgrado". (Shaver, 2012, p.2-3)

En este contexto, es relevante articular una herramienta de sistematización de información que posibilite mejorar la relación con el cliente y así recolectar información de interés para apostarle a la creación de propuestas personalizadas o ya bien el descubrimiento de nuevos nichos de mercado, pues ahora "los clientes dicen a las empresas qué tipo de productos o servicios desean y cuándo, dónde y cómo quieren comprarlos". (Kotler y Keller, 2012, p.15) contribuyendo directamente al mejoramiento de la relación de la marca con el cliente y a la toma de decisiones estratégicas de la oferta de las organizaciones de educación superior. Esta herramienta se denomina Sistema de Información de Mercadeo.

\section{Sistemas de Información de Mercadeo - SIM -}

Los Sistemas de Información de Mercadeo son una herramienta donde interactúan personas, equipos de cómputo y procedimientos definidos que permiten la recopilación, almacenaje y manipulación de información organizacional externa e interna con fines direccionales y de control correspondiente. (Romeiro y Garmendia, 2007) (Kotler y Keller, 2012) (Hernández, 2003) Su nacimiento se debe al "incremento de la competencia en los mercados de bienes y servicios, la rapidez en el desarrollo de las tecnologías de información, el aumento de la incertidumbre en el entorno y la reducción de los ciclos de vida de los productos" (Hernández, 2003, p.1).

A lo largo de los años, han sido infinitas las investigaciones que se han realizado en pro de la fusión entre información (datos) y tecnología (software) para generar y respaldar la toma de decisiones gerenciales basados en datos reales. La información se convirtió en un elemento clave para la gestión y la falta del "modelo es un déficit necesario de superar, ya que una comprensión del uso de la administración efectiva y responsable de la información, es importante para todos aquellos trabajadores del conocimiento empresarial en la sociedad de la información global de hoy". (Romeiro y Garmendia, 2007, p. 600) 
(Hernández, 2003) Es de suponer que los SIM desempeñan un papel esencial en el ejercicio de las operaciones eficientes; favoreciendo la gerencia efectiva y el éxito estratégico empresarial.

No obstante, el camino de implementación de un Sistema de Información de Mercadeo no es fácil, y dentro del aprendizaje, las empresas deben contemplar variables adecuadas, precisas y de mayor importancia de recopilación que contribuyan al crecimiento organizacional, y no por el contrario que entorpezcan los procesos y torne más lento el sistema en su totalida. (Kotler y Keller, 2012)

El SIM es una herramienta conformada por subsistemas, cajas de información instaladas dentro de la organización capaces de recibir información, almacenar los datos para su disposición inmediata, procesar eficazmente y sin errores y proporcionar seguridad para evitar la pérdida de información. Todo esto con el fin de entregar un "insumo revelación" que respalde directrices institucionales. "Las empresas con mejor nivel de información tienen más oportunidades de elegir correctamente sus mercados, desarrollar ofertas más adecuadas y ejecutar una planificación de mercadeo más certera". (Kotler y Keller, 2012, p.69) (Hernández, 2003) Sin embargo, de nada sirve el esfuerzo de tiempo y dinero invertido por las organizaciones, si no se cuenta con una cultura organizacional de orden y respeto por el SIM mismo y por el conocimiento del cliente interno y de su importancia como ente transformador. El éxito del SIM requiere compromiso y claridad con su implementación, no solo como una herramienta, sino como un modelo de gestión empresarial. (Romeiro y Garmendia, 2007)

\section{Método}

Esta investigación tiene un enfoque cualitativo-exploratorio sustentado en la revisión bibliográfica de antecedentes encontrados en bases de datos académicas como Scopus y Procuest.

Para la construcción del marco teórico y la obtención de resultados, se planteó una estrategia de recolección de información definiendo un paquete de ecuaciones de búsqueda que cumplieran con características definidas como la inclusión del tema investigado, tiempo de la publicación, áreas de enfoque del desarrollo investigativo y que estuvieran escritas en inglés o español.

En total se descargaron 78 documentos compuestos por artículos científicos, tesis doctorales y de maestría que cumplieran con los requerimientos antes nombrados. Todos los documentos aportaron directa o indirectamente en la escritura de este artículo y por tal motivo se consideró importante su referencia.

Para el análisis efectivo de toda la información recolectada, se diseñó un instrumento apropiado de clasificación y almacenamiento de información denominado fichas investigativas. Estas fichas, consignan la información resumida de todos los documentos y posibilitan un análisis posterior práctico y ágil de toda la información; obteniendo como resul- 
tado un esquema visible de los conceptos y datos relevantes descritos en los documentos que son de interés principal para la elaboración de este artículo.

\section{Resultados}

La incertidumbre y fluctuación de las necesidades del mercado a las que las empresas a nivel mundial se enfrentan, sugieren la necesidad de un cambio y restructuración organizacional en el que se plantee una respuesta a la medida de las necesidades del consumidor. El sector educativo es uno de los mercados más afectados con esta nueva dinámica empresarial llevándolo a la trasformación de su tradicional estrategia de mercadeo en pro de atraer clientes potenciales, retener los existentes y diferenciarse dentro de la saturación de oferta existente. Propuestas como la orientación al mercado, la gestión de marca y programas de relacionamiento con el cliente -CRM - son los cambios evidenciados en las prácticas de mercadeo de las universidades consultadas para esta investigación.

\section{Orientación al Mercado (OM)}

Para Kotler y Armstrong citado por José Sixto Garcia, el mercadeo del siglo XXI "Se centra en las necesidades del público objetivo y en proporcionarle satisfacción competitiva y rentable" (García, 2010 , p.74) por ello, definen que el mercadeo, "se apoya en cuatro pilares fundamentales: la definición del mercado, la orientación al mercado, la coordinación de mercadeo y la rentabilidad" (García, 2010, p.74) De igual manera, Garcia, especifica que el mercadeo actual "hace clientes y no productos mediante la satisfacción de sus necesidades a través de una actuación de mercadeo integrado que obtiene beneficios" (García, 2010, p.74)

En los últimos decenios, investigaciones dedicadas a la aplicación de la orientación al mercado solo se han centrado en empresas manufactureras; sin embargo, hoy en día se han ido vinculando a otros sectores productivos y de servicios como el de salud y el de educación dado que se ha demostrado que la orientación al mercado influencia positivamente en el desempeño económico de las empresas. (Rivera-Camino y Ayala, 2010; Narver y Slater, 1990, citado en Lombao, 2006; Kohli, Jaworski y Kumar, 1993, citado en Lombao, 2006)

Aunque la evidencia empírica para apoyar estas afirmaciones es insuficiente, "las organizaciones saben que tienen que desarrollar una orientación hacia el mercado" (Lings y Greenley, 2009, p.2) para desarrollar niveles "altamente satisfactorios de respuesta a los retos del entorno con calidad y pertinencia”. (Lombao, 2006, p.63)

Para Mary Carmen Lombao "Un enfoque de Orientación al Mercado implica el conocimiento de las necesidades presentes y futuras del consumidor, los factores exógenos y la coordinación interna de procesos para dar respuesta a las demandas" (Lombao, 2006, p.64); Por ello, las universidades hoy en día, aplican diferentes estrategias de mercadeo eficaces en el mundo de los negocios manufactureros en un intento por mejorar la relación de la marca con sus 
clientes; tomando la orientación al mercado como un factor estratégico integrado a toda la organización, fundamental en la toma de decisiones.

Pero, ¿Qué significa una Orientación al Mercado en el contexto del mercadeo educativo? Para responder esta pregunta, hay que partir de la definición inicial; para Rajan Varadarajan y Satish Jayachandran (1999, citado por Lombao, 2006, p.65) la OM es un "conjunto de acciones que asumen las organizaciones, así como una cultura distintiva que las dota de capacidades para descubrir la demanda, anticiparla y responder a las variaciones de los mercados", y es también una "filosofía empresarial de gestión integral, una cultura organizativa denominada cultura de mercadeo que contempla una perspectiva de comportamiento coordinado en función del tiempo" (González, Vijande y Casielles, 2001, p.3)

Esta filosofía plantea su aplicación definiendo indicadores de gestión trasversales a las diferentes áreas administrativas de la organización, desde donde se evalúa constantemente el funcionamiento de la unidad de negocio "para que las funciones se desempeñen bajo una sintonía común de captar, procesar, difundir y responder inteligentemente al mercado" (Lombao, 2006, p.66; Varadarajan y Jayachandran, 1999; Varela, Gutiérrez y Antón, 1998, citado en González, Vijande y Casielles, 2001)

En tal sentido, las universidades intentan convertir en oportunidades las exigencias del entorno, y en función de la adaptación a esa filosofía adoptan modelos que permiten facilitar y articular la gestión estratégica de las unidades en conjunto con la Orientación al Mercado. Dentro de los grandes exponentes de estos modelos de adaptación se encuentran investigadores de gran categoría en el tema como, Shapiro, Ruekert, Diamantopoulos y Hart, Pelham y Wilson, Deng y Dart, Cadogan, Lambín Tuominen y Möller, Homburg y Becker entre otros. (González, Vijande y Casielles, 2001; Rivera-Camino y Ayala, 2010)

En el año 2006, en la Universidad Metropolitana de Venezuela, se realizaron exploraciones empíricas de la aplicación del modelo de adhesión de Orientación al Mercado en la educación superior que plantean Kohli y Jaworski (citados por González, Vijande y Casielles, 2001); aunque no se especifican los resultados, se exponen a continuación el modelo aplicado.

La aceptación de una filosofía de negocio permite a la organización la satisfacción de sus clientes, esta se sustenta en tres principios: (1) la orientación primaria a las necesidades actuales y futuras del cliente, orientación que deberá venir acompañada de la valoración de los factores de mercado que puedan afectar la satisfacción de las necesidades y preferencias del mismo; (2) la integración y coordinación de las actividades de mercadeo entre las distintas funciones empresariales, con la vista puesta en el cliente; y (3) la consecución de una rentabilidad que recompense el esfuerzo realizado, si bien, matizan, se considera esta rentabilidad más 
como una consecuencia de estar orientados al mercado que como un fin en sí mismo. (González, Vijande y Casielles, 2001, p.5)

Por otro lado, Lombao (2006) demostró que en el mercadeo del sector educativo la relación con el entorno representa un elemento substancial para la transformación y la adopción adecuada de la filosofía (OM), además, logró concluir que la satisfacción del cliente es un elemento esencial del éxito de las organizaciones de servicio y que para facilitar la adopción de este enfoque, existen dos factores inherentes-no excluyentes.

Fomentar una creencia generalizada en la gerencia de la conveniencia de estar orientados al mercado y la creación de una unidad de mercadeo con el rango de Dirección, que no debería visualizarse como responsable única...pues todos los miembros de la organización lo son. (Lombao, 2006, p.81)

Entonces, la Orientación al Mercado está catalogada por los analistas como una herramienta de gestión organizacional enfocada al mercado que en conjunto con el compromiso ineludible de los organismos directores y el desarrollo óptimo de sus funciones y la de todo el plantel empresarial, aportan directamente al éxito de comercialización de cualquier producto o servicio.

\section{El Customer Relationship Manage- ment (CRM)}

La lógica de los negocios actuales afecta directamente a las organizaciones a nivel mundial, incluyendo también al sector educativo, demandando cada vez más una relación cercana con sus clientes. "Cada vez existe menor diferenciación a nivel de los productos y servicios que se ofrecen, de esta forma las empresas se han vuelto más vulnerables a una competencia de menores precios" (Di Tommaso, 2005, citado en Petrella, 2008, p.10) Por tal motivo, las Universidades están gestando nuevas soluciones a los problemas planteados por el cambio constante del mercado y la migración del mercadeo transaccional de los años 50 al mercadeo relacional de la actualidad.

Tradicionalmente, las empresas han concentrado sus esfuerzos de mercadeo en la captación de nuevos clientes y maximización de los beneficios...Sin embargo, las empresas orientadas a los servicios se han alejado de esta estrategia de mercadeo tradicional hacia un enfoque de mercadeo relacional que se centra en el desarrollo de relaciones a largo plazo con los clientes existentes. (Fontaine, 2014, p.106)

Mientras que el mercadeo transaccional resultó efectivo para las empresas manufactureras, "las empresas orientadas a los servicios, que tratan personalmente con el cliente, encontraron un enfoque relación-comercialización más rentable" (Ackerman y Schibrowsky, 2007, citado en Shaver, 2012, p.14) pues los clientes demandan más que el préstamo de un simple servicio y por ello proporcionan mayor fidelidad a las empresas que complementan sus servicios básicos, por lo que la relación clienteempresa y retención se convirtieron en factores de importancia para la su- 
pervivencia de las empresas de servicios. (Trustrum y Wee, 2007, citado en Shaver, 2012)

El mercadeo relacional es más que un nuevo modelo, es un nuevo paradigma, "se basa en la premisa de aprender todo lo relevante sobre el cliente y luego usar esa información para servirles. Es una estrategia de retención de clientes ampliamente aceptada y practicada en la comunidad empresarial" (Shaver, 2012, p.15) Los beneficios inherentes a la aplicación de la estrategia del CRM con el cliente son muchos, entre ellos, "reducción de costos, publicidad gratuita a través del voz a voz, y el valor de tiempo de vida de un cliente" (Shaver, 2012, p.15)

Pero, ¿Qué es en sí el CRM? Este instrumento, se define a grandes rasgos como la capacidad de una empresa para obtener clientes, conocerlos, mantenerse en comunicación con ellos y garantizar su conservación; pero sobre todo, se define como una herramienta que permite generar productos personalizados, segmentación del mercado, nuevos servicios y productos, y mayor receptividad y satisfacción del cliente. (Swift, 2002)

Según Morgan y Hunt (1994) el CRM son "todas las actividades de mercadeo enfocadas en el establecimiento, desarrollo y mantenimiento de los intercambios relacionales exitosos" (Morgan y Hunt, 1994, p.22) entre las empresa y sus clientes. Otros autores complementan esta visión afirmando que "el CRM es una actitud, un enfoque, un conjunto de prácticas dirigidas a los clientes, que una vez que forman parte de la cultura organizacional, facilitan la integración de una herramienta de software" (Petrella, 2008, p.5) para su administración y control; pero, aun así, "lo que debe quedar claro es que no necesariamente se hace CRM porque se compra un software y se implementa bien desde el punto de vista de tecnología" (Petrella, 2008, p.5) Como plantea Barton Goldemberg citado por Carlos Petrella "El último gran desafío de la industria gira en torno a la mejor manera de integrar CRM en los sistemas legados de la compañía y la infraestructura tecnológica relacionada”. (Petrella, 2008, p.10-11)

Robert Ackerman y John Schibrowsky (2007) creen que el futuro de la educación superior está en la construcción de relaciones a largo plazo con los estudiantes y sostienen que:

En esencia, todo el mundo es un gestor de retención; mientras que los empleados de primera línea son importantes, un programa de mercadeo y relación exitosa requerirá compromiso administrativo de alto nivel viendo la construcción de relaciones como una inversión a largo plazo del negocio en lugar de un gasto operacional. (Ackerman y Schibrowsky, 2007, p.328)

Los investigadores contemporáneos discuten las ventajas que representa aplicar los principios del CRM a la industria de la educación superior, pero es claro que la retención de clientes -en este caso estudiantes- se está convirtiendo cada vez más en un reto mayor para los empresarios. (Fontaine, 2014) 


\section{Gestión y Posicionamiento de Marca}

Las IES (Institución de Educación Superior) se están moviendo hacia un modelo de corporación y de comercialización agresiva de sí mismas. (Katz et al., 1999; Naudé y Ivy, 1999; Pusser, 2002; Ruch, 2001, citado en Williams y Omar, 2014) "En el presente, se enfrentan a retos cada vez más complejos. En particular, su entorno operativo está experimentando grandes transformaciones, incluyendo el cambio de los patrones de demanda y la intensificación de la competencia global". (Kallio, 1995; Jarvis, 2000; Gibbs, 2001; Veloutsou, Lewis y Paton, 2004, citados en Simões y Soares, 2010, p.371)

Mientras los estudiantes adopten un enfoque consumista de toma de decisiones y evalúen constantemente el retorno de la inversión, las IES tendrán que evaluar frecuentemente el posicionamiento de su marca en el mercado, pues según investigaciones, este es uno de los ítems protagonistas entre los jóvenes para seleccionar el ingreso a una institución u otra. (Cobb, 2001; Koku, 1997; Morphew et al, 2001; Nguyen y LeBlanc, 2001; Sevier, 2002; Toma, Dubrow y Hartley, 2005; Williams, 2012; Williams, Osei y Omar, 2012, citados en Williams y Omar, 2014)

No obstante, la interpretación del posicionamiento, gestión y reputación de la marca en una IES se puede analizar "diferente a otras industrias de servicios, en parte, ya que los empleados juegan un papel decisivo en la construcción de la reputación de una IES dándole alma a la marca". (Heaney y Heaney,
2008; Hemsley-Brown y Oplatka, 2006; Lowrie, 2007; Williams, 2012, citados en Williams y Omar, 2014, p.224)

La educación superior es una industria particular, se involucran acciones en gran medida intangibles, con características que incluye un enfoque a las personas y una búsqueda constante de la creación de una larga e incesante relación con el cliente. (Williams y Omar, 2014) "las IES debe prestar atención a los aspectos de intangibilidad y la inseparabilidad de los servicios educativos. Como una marca de servicio, las IES requieren mayor énfasis en la comercialización interna, en parte, porque todos los empleados se convierten en puntos de contacto de los consumidores" (Berry, 2000, citado en Williams y Omar, 2014, p.224)

Pero, ¿Qué hace importante el posicionamiento y gestión de marca de una IES en el mercado? Danilo, Robert y Maktoba responden esta pregunta afirmando que "Las marcas son esenciales a la condición social de los consumidores, y, de hecho, la obtención de un título de educación superior es el otorgamiento de un cierto nivel de estatus social". (Hamann, Williams, y Omar, 2007, citados en Williams y Omar, 2014, p.224) De ahí que las IES busquen incesantemente posicionarse en lo más alto de los rankings a nivel internacional, pues en coherencia con lo anterior, esto le brindará no solo estatus social y económico al consumidor final, sino también un reconocimiento adicional para la IES afianzando la imagen de calidad y prestigio. Igualmente, la literatura que habla sobre el tema, anota además 
que la auditoria de marca es pertinente al tema de posicionamiento y gestión de marca, pues monitorear su estado constante en el mercado posibilita una reacción adecuada en el momento requerido previniendo el declive de la marca en la mente de los consumidores. (Williams y Omar, 2014)

Todas las organizaciones de Educación Superior, toman decisiones frente a su marca cuando los objetivos deseados de rentabilidad, comercialización o adaptabilidad no son alcanzados con éxito. De ahí, que las empresas inicien una auditoría interna en búsqueda del proceso o procesos que representan el punto de quiebre de toda la gestión de la organización. "El valor de la marca debe ser gestionado de forma activa en el tiempo mediante el refuerzo de la misma y, en algunos casos si es necesario, mediante la revitalización de ella". Para las IES ejecutar una auditoría de marca representa "Un proceso de toma de decisiones estratégicas y de deliberación que lleva a una conclusión de marca: retirarse, revitalizar, reorientar o cambiar el nombre" (Williams y Omar, 2014, p.226)

La construcción de marca para las IES se está convirtiendo en un objetivo estratégico; sin embargo, en la ausencia de modelos claros de gestión de marca para este segmento, se debe tener especial cuidado pues a veces estas, "resuelven sus problemas inmediatos, otras veces el crecimiento del mercado silencia la gravedad de los problemas". (Williams y Omar, 2014, p.237)

Hay muchos caminos para gestionar una marca, por ello, las IES deben de- sarrollar estrategias de sostenibilidad de marca a largo plazo interna y externamente, y no instaurar estrategias temporales de respuesta, pues este tipo de enfoques ofrecen beneficios a corto plazo, y tienden a esfumarse. "Cuando las comunicaciones y los profesionales de mercadeo desarrollan estrategias de marca que no se admiten internamente en las organizaciones, los consumidores se sienten traicionados y frustrado" (Whisman, 2009, p.367) por esa razón, es de suma importancia que cualquier modificación o mejora que se le aplique a una marca, debe ser primero adaptada al interior de la organización y adoptarla como una identidad corporativa donde el tono de comunicación y acción sea el mismo hacia el interior de la organización como hacia el exterior de la misma. Generalmente todos los cambios o estrategias de mejoramiento de marca estudiados en los aportes exploratorios de los diferentes autores para esta investigación, se enfocan en realizar respuestas de adentro hacia afuera, y no internamente, propiciando una desarticulación del principio rector que debe asumir cualquier organización.

\section{Conclusiones}

- Actualmente, las Universidades alrededor del mundo, no cuentan con una estructura definida de Mercadeo que soporte la comercialización eficiente y responsable del servicio educativo.

- La iniciativa que proponen algunos autores en la trasformación del modelo tradicional del marketing mix para los servicios, es integrar al cliente dentro de la ecuación de variables influyentes. 
- Para mejorar la comercialización del servicio educativo no basta solo con aplicar una de las tres alternativas planteadas en este artículo; los autores afirman que es fundamental permear la estructura organizacional con un pensamiento de eficiencia, calidad y cuidado de marca.

- Desde la información analizada para el desarrollo de esta investigación no fue posible determinar el uso del SIM como sistema de recolección, almacenamiento y distribución de información. No obstante, el CRM, la Orientación al Mercado, y la Gestión de Marca, se perfilan como las herramientas de mercadeo principales a tener en cuenta para diseñar un modelo de gestión de mercadeo para el sector educativo, pues al no ser evidente la clasificación o identificación precisa de un dato específico utilizado por las organizaciones para la toma de decisiones estas herramientas esbozan un camino posible para potencializar, promover e inclusive intentar comprender el deber ser del mercadeo específico de los servicios de educación superior.

\section{Referencias}

Ackerman, R., \& Schibrowsky, J. (2007). A Business Marketing Strategy Applied to Student Retention: A higher Education Initiative. Journal of College Student, 9(3), 307-336.

Arango, X. C. (2009). Ministerio de Educación Nacional República de Colombia. Obtenido de: www.mineducacion.gov.co/1621 /articles-230245_archivo_pdf _articulo_internacionalizacion.pdf

Barnes, J. (1994). Close to the customer: But is it really a relationship? Journal of Marketing Management, 561-570.

Berry, L. (2000). Cultivating service brand equity. Journal of the Academy of Marketing Science, 28(1), 128-137.

Caetano, G. (2003). Marketing en los servicios de Educación: Modelos de percepción de calidad. (Tesis de Doctorado). Universidad Complutense de Madrid, Madrid, España.

Cobb, C (2001). The concept of brand identity in relation to student's intent-topersist. (Tesis de Doctorado) University of Oklahoma, Oklahoma, USA

Colombia. Congreso de la República. Ley 30. (28, diciembre, 1992). Por el cual se organiza el servicio público de la Educación Superior. Diario Oficial. Bogotá.

Fontaine, M. (2014). Student Relationship Management (SRM) in Higher Education: Addressing the Expectations of an Ever Evolving Demographic and Its Impact on Retention. Education and Human Development, 3(2), 105-119.

García, J. S. (2010). El Marketing y su origen a la orientación social: desde la perspectiva económica 
a la social. Los aspectos de organización y comunicación. Em Questão, 16(1), 61-77.

Gibbs, P. (2001). Higher education as a market: A problem or solution? Studies in Higher Education, 26, 85-94.

González, L. I., Vijande, M. L., \& Casielles, R. V. (2001). Universidad de Oviedo. Obtenido de http://dialnet.unirioja.es/servlet /articulo? codigo $=1252647$

Hamann, D., Williams, R., \& Omar, M. (2007). Branding strategy and consumer high technology product. The Journal of Product \& Brand Management, 16(2), 98111.

Hemsley-Brown, J., \& Oplatka, I. (2006). Universities in a competitive global marketplace: A systematic review of the literature on higher education marketing. International Journal of Public Sector Management, 19(4), 316338.

Hernández, A. (2003). Los Sistemas de Información: Evolución y desarrollo. Proyecto social: Revista de Relaciones laborales, (10-11), 149-165.

Jarvis, P. (2000). The changing university: Meeting a need and needing to change. Higher Education Quarterly, 54, 43-67.

Kallio, R. E. (1995). Factors Influencing the College Choice Decisions of Graduate Students. Research in Higher Education, 36(1), 109124.
Katz, R., Blustain, H., Duderstadt, J., Farrington, G., Goldstein, E., Graves, W., \& Lozier, G. (1999). Dancing with the devil: Information technology and the new competition higher. San Francisco: Jossey-Bass.

Kohli, A. K., Jaworski, B. J., \& Kumar, A. (1993). MARKOR: A Measure of Market Orientation. Joumal of Marketing, 30(4), 467-477.

Koku, P. S. (1997). What is in a name? The impact of strategic name change on student enrollment in colleges and universities. Journal of Marketing for Higher Education, 8(2), 53-71.

Kotler, P., y Keller, K. (2012). Dirección de Marketing. (14 Ed.). México: Pearson.

Laval, C. (2004). La Universidad no es una empresa. Barcelona: Paidós.

León, G. (2004). La educación en el contexto de la educación. Revista Historia de la Educación Latinoamericana, 343-354.

Lings, I., \& Greenley, G. (2009). The impact of internal and external market orientations on firm performance. Journal of Strategic Marketing, 17(1), 41-53.

Lombao, M. C. (2006). La Orientación al Mercadeo en la gestión Universitaria. Anales de la Universidad Metropolitana, 6(1), 61-85.

Lowrie, A. (2007). Branding higher education: Equivalence and difference in developing Identity. Jour- 
nal of Business Research, 60(9), 990-999.

Mateus, J. R., \& Brasseet, D. W. (2002). La globalización: sus efectos y bondades. Economía y Desarrollo, 65-77.

Ministerio de Educación Nacional República de Colombia. (20 de Julio de 2009). Ministerio de Educación Nacional República de Colombia. Obtenido de www.mineducacion.gov.co/1621 /article-196472.html

Morgan, R. M., \& Hunt, S. D. (1994). The Commitment-Trust Theory of Relationship Marketing. The Journal of Marketing, 58(3), 2038.

Naranjo, C. (2011). Marketing educativo; desarrollo de una estrategia C.E.M. aplicado a la Universidad nacional de Colombia sede Manizales como base para la fidelización de clientes y complemento a la estrategia de C.R.M. (Tesis de Maestría). Universidad Nacional de Colombia, Manizales, Colombia.

Narver, J., \& Slater, S. (1990). The effect of a Market Orientation on Business Profitability. Journal of Marketing, 54, 20-35.

Naudé, P., \& Ivy, J. (1999). The marketing strategies of universities in the United Kingdom. The International Journal of Educational Management, 13(3), 126-134.

Nguyen, N., \& LeBlanc, G. (2001). Image and reputation of higher edu- cation institutions in students' retention decisions. International Journal of Educational Management, 15(6), 303-311.

Ospina, M., y Sanabria, P. (2010). Un enfoque de mercadeo de servicios educativos para la gestión de las organizaciones de educación superior en Colombia: el modelo MIGME. Revista de la Facultad de Ciencias Económicas: Investigación y reflexión, 18(2), 107136.

Petrella, C. (2008). Gestión de la relación de las universidades con docentes, estudiantes y egresados. Revista Iberoamericana de Educación, 47(5), 1-14.

Ponce, J. (2008). Un Nuevo Marketing de las Universidades. Objetivos formativos y demandas sociales. Recuperado de http://dspace.uah.es/dspace/ handle/10017/1461

Pusser, B. (2000). The role of the state in the provision of higher education in the United States. Australian Universities Review, 43(1), 24-35.

Real Academia Española. (2015). Real Academia Española. Obtenido de http://lema.rae.es/drae/srv/ search?id=r1Qn4oKX7DXX2u XRACx 1

Rivera-Camino, J., \& Ayala, V. M. (2010). Market orientation at universities. Construct and exploratory validation. Innovar, 20(36), 125-138. 
Romero, A. (2002). Globalización y pobreza. Pasto: Unariño.

Romeiro, J., y Garmendia, F. (2007). Sistemas de Información de Marketing SIM: Más que simples cajas tecnológicas. Esic, 128, 95107.

Ruch, R. (2001). Higher Ed, Inc.: The rise of the for-profit university. Baltimore: The Johns Hopkins University Press.

Shaver, B. J. (2012). Meeting undergraduate students' expectations of the university experience: How enrollment managers can secure students' loyalties (Tesis de Doctorado) University of California, San Diego-California State University, San Marcos, California, Estados Unidos de América

Simões, C., \& Soares, A. M. (2010). Applying to higher education: information sources and choice factors. Studies in Higher Education, 35(4), 371-389.

Swift, R. S. (2002). CRM: Como mejorar las relaciones con los clientes. México: Pearson.

Théry, J. (2013). National Geographic España. Obtenido de www.nationalgeographic.com.es /articulo/historia/grandes_reportajes/8629/nacimiento_universidad.html

Toma, J., Dubrow, G., \& Hartley, M. (2005). The uses of institutional culture: Strengthening and building brand equity in higher education. Higher Education Report, 31(2), 1-105.

Universidad de León. (2012). Universidad de León. Obtenido de http://www.unileon.es/estudiantes/internacional-movilidad/plan-de-internacionalizaci $\% \mathrm{C} 3 \% \mathrm{~B} 3 \mathrm{n}$

Varadarajan, R., \& Jayachandran, S. (1999). Marketing Strategy: An Assessment of the State. Academy of Marketing Science, 27(2), 120-143.

Varela, J., Gutierrez, A., \& Antón, C. (1998). La cultura de orientación al mercado y otros antecedentes seleccionados deln rendimiento empresarial. Un contraste de Metodologías. X Encuentros de Profesores Universitarios de Marketing, 135-139.

Veloutsou, C., Lewis, J., \& Paton, R. (2004). University selection: Information requirements and importance. International Journal of Educational Management, 18,160-171.

Wang, X. (2009). Institutional recruitment strategies and international undergraduate student university choice at two canadian universities. (Tesis de Doctorado) University of Toronto, Toronto, Canada

Whisman, R. (2009). Internal branding: a university's most valuable intangible asset. Journal of Product \& Brand Management, 18(5), $367-370$. 
Williams, R. L. Jr. (2012). Branding through renaming for strategic alignment in service organizations. (Tesis Doctorado). Edinburgh Napier University, Edinburgh, Escocia.

Williams, R. L., \& Omar, M. (2014). How branding process activities impact brand equity within Higher Education Institutions. Journal of Marketing for Higher Education, 24(1), 1-10.

Williams, R. L., Osei, C., \& Omar,
M. (2012). HEI branding as a component of country branding in Ghana: Renaming Kwame Nkrumah university of science and technology. Journal of Marketing for Higher Education, 22(1), 71-81.

Williams, R., \& Omar, M. (2014). Applying brand management to higher education through the use of the Brand Flux Model ${ }^{\mathrm{w}}-$ the case of Arcadia University. Journal of Marketing for Higher, 24(2), 222-242 